\title{
Exploring the Rescue Strategy for Cardiac Arrest in Makeshift (FangCang) Hospital Workers during the Pneumonia Outbreak Associated with COVID-19
}

\author{
*Ke FENG ${ }^{1}$, Bin $\mathrm{MEI}^{2}$, Zhongwei CHEN ${ }^{1}$, *Xufeng $\mathrm{FU}^{3}$ \\ 1. Department of Emergency, General Hospital of Ningxia Medical University, Yinchuan, Ningixa 750004, China \\ 2. Department of Neurology, Zhongnan Hospital of Wuhan University, Wuban, Hubei 430071, China \\ 3. Key Laboratory of Fertility Preservation and Maintenance of Ministry of Education, Ningxia Medical University, Yinchuan \\ 750004, China
}

*Corresponding Authors: Emails: fengkedoct@163.com; fuxufeng100@163.com

(Received 11 Mar 2020; accepted 19 Mar 2020)

\begin{abstract}
Background: Beginning in Dec 2019, a novel coronavirus, designated SARS-CoV-2, has caused an international outbreak of respiratory illness termed COVID-19. The workers in the FangCang hospital have to work for more than $8 \mathrm{~h}$ and the work is high strength. Furthermore, to protect health and prevent serious cross-infection, they need to wear isolation equipment when working in FangCang hospital. These characteristics increase the risk of cardiac arrest (CA), which seriously endangers the lives of workers.

Methods: We participated in the rescue of the patient and workers at first-line in FangCang hospital, and summarized the rescue strategies for workers rescuing.

Results: Workers with CA were rescued in time according our guideline and showed zero dead in FangCang hospital.

Conclusion: This study establishes the strategy for the CA of workers including the establishment of an inFangCang resuscitation team, the establishment of a dedicated rescue room, and the formulation of rescue measures and procedures for CA of workers in the FangCang hospital. Therefore, we aimed to provide a strategy for the rescue of workers with CA in the FangCang hospital and share the success in rescuing with the world.
\end{abstract}

Keywords: COVID-19; Workers; Cardiac arrest; China

\section{Introduction}

In Dec 2019, a series of pneumonia patients infected with an unidentified coronavirus have appeared in Wuhan, Hubei Province, central China. WHO has officially named the disease COVID19 (1). The new type of coronavirus has a strong transmission ability from person to person, long incubation period and asymptomatic carrier transmission (2). The epidemic situation spread rapidly in a short time. Up to Mar 22, the number of confirmed cases exceeded 300,000, affecting nearly 120 countries and regions, 81,054 in China 67,800 were confirmed cases in Hubei Province (50005 in Wuhan). The COVID-19 was announced by the WHO as "an international public health emergency of concern" on Jan 30, 2020 (3). The large spread epidemic, the wide cumula- 
tive area and the much number of infected people determine the admission is difficult. In order to effectively treat patients and control the source of infection on time, the government urgently set up nearly 20 makeshift (FangCang) hospitals in mid-early February 2020, which played a vital role in the overall epidemic prevention and treatment. Cardiac arrest (CA) is the sudden termination of cardiac ejection due to various reasons, and severe ischemia, hypoxia and even death of important organs (4). The main reasons that the workers are likely to induce CA during work are: 1) to attain careful protection and prevent crossinfection, workers need to wear isolation equipment to work continuously for 6-8 h or even longer. The high-intensity and long-term work are likely to induce hypoxia, water and electrolyte disorders, Hypoglycemia, etc. 2) A lot of workers have basic diseases such as hypertension, diabetes, and heart and lung diseases.

\section{Methods}

In order to ensure that the workers entering the FangCang Hospital could get helpful treatment, and prevent and reduce the chance of being infected after being exposed to other medical workers participating in the rescue, we combined with this personal participation in the experience of the Wuhan FangCang hospital's epidemic treatment work, discussed and revised the FangCang Emergency rescue strategy for CA in hospital workers.

\section{Results and Discussion}

We summarized the successful experiences and puts forward the corresponding strategies as follows, and the flow chart of treatment is showed in Fig. 1.

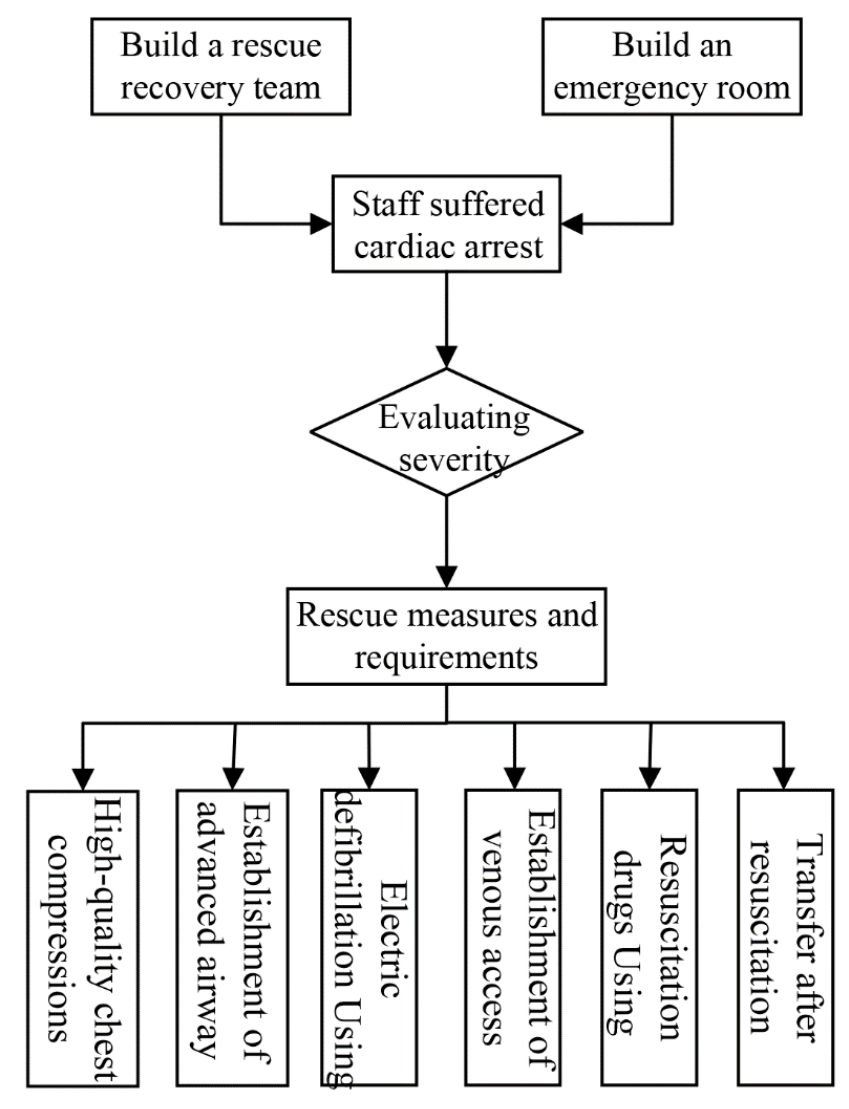

Fig.1: Flow chart of treatment for cardiac arrest of worker in FangCang Hospital, China 


\section{Establish an in-FangCang recovery team}

The team leader is a senior physician engaged in emergency and critical medicine majors attended the American Heart Association (AHA) training and obtained a qualified certificate. The number of team members to meet the minimum number of resuscitation measures, generally, 3 people, and the members should have experienced nursing experience, preferably engaged in critical care professional nursing workers. The team leader is responsible for the assessment and judgment of the condition, directing rescue, establishing advanced airways, and transferring patients. One member is responsible for chest compression, and the other member is responsible for operating the monitor or defibrillator, establishing drug delivery channels, and giving resuscitation drugs (5). Two members exchange their roles every two minutes.

\section{Establish a rescue room in the Fang Cang}

To minimize the risk of nosocomial infections, rescue operations should be avoided in an open environment. According to the layout of the FangCang and the workers' distribution area, a rescue room for workers in the FangCang is specially established. The rescue room is relatively closed and managed by the nursing team. The rescue room should be regularly disinfected, equipped with defibrillators, breathing airbags, tracheal intubation, mechanical resuscitation devices, monitors, oxygen sources, rescue drugs, transport ventilators, and establish venous access, and other rescue items. The emergency room is kept in a standby state for $24 \mathrm{~h}$, and if there is an emergency, it will be immediately transferred to the emergency room of the ward for rescue.

\section{Develop rescue measures and procedures Condition evaluation}

If a worker member suddenly feels unwell during work and suspects a sudden CA, the leader of the resuscitation team should immediately organize the resuscitation team to transfer the patient to the rescue room in the FangCang. First, the team leader quickly unfastened the protective clothing to check the consciousness, carotid pulsation, and assessment of breathing conditions, but the mask should be reserved to prevent crossinfection in the hospital. Next, the member determines whether it is a CA. According to clinical symptoms, other physical discomforts caused by hypoglycemia, hypotension, syncope, acute pain, and high tension caused by excessive working hours in the FangCang should be excluded.

\section{Rescue measures and requirements}

Members of the FangCang resuscitation team should quickly place the worker in a proper position for a sudden CA. Place the rescued worker on a flat floor or a hard bed, perform chest compressions under the command of the team leader, remove their masks at the same time, keep their airways open, and provide airbag ventilation. If necessary, immediately intubate and conduct electrical Defibrillation, establish venous access, and give rescue medication as required.

\section{High-quality chest compressions}

High-quality chest compressions are the key to successful cardiopulmonary resuscitation (CPR). Chest compressions must be performed as soon as possible after CA, and the quality of the compressions must be guaranteed. High-quality chest compressions include a compression speed of 100-120 times per minute, a compression depth that causes the sternum to sink $5-6 \mathrm{~cm}$, a complete rebound of the chest after each compression, and minimizing the interruption of chest compressions (6). Chest compressions are an extremely labor-intensive task. The protective clothing worn by members of the CPR team has limited comfort, and rescuers are prone to fatigue. In order to prevent the rescue workers from being infected after being exposed during the rescue process, the number of CPR team members is strictly limited, therefore, it is recommended to use mechanical resuscitation devices during CPR. The results of the current study showed that mechanical resuscitation devices do not improve patient outcomes compared to unarmed chest compressions (7). In the special environment for rescue of new coronavirus pneumonia, the mechanical resuscitation device can play a unique advantage. 
Establishment of advanced airways

The establishment of advanced airways during CPR is performed by an experienced and capable resuscitation team leader to ensure that the advanced airways are placed correctly while shortening the operation time to reduce the interruption of chest compressions during the operation (8). Patients with CA due to factors such as hypoxia should consider establishing an advanced airway as soon as possible. Compared with balloon mask ventilation, the establishment of advanced airways for artificial ventilation can provide better ventilation and ventilation function support, which is conducive to the drainage of respiratory secretions and prevents aspiration (9). When using a conventional laryngoscope to establish an advanced airway, the operator's face must be close to the patient's mouth to facilitate direct observation, which increases the risk of infection for medical workers (10). To reduce the risk of exposure, it is recommended to use visualization equipment to place advanced airways as a priority.

\section{The use of electric defibrillation}

When a shockable rhythm (ventricular fibrillation or pulseless ventricular tachycardia) occurs in the worker's CA, electrical defibrillation is the most effective rescue method. Electrical defibrillation should be implemented as soon as possible, and manual defibrillation should be used first. During the defibrillation function, the time required to analyze the heart rhythm is short. During the defibrillator charging process, team members can continue to perform chest compressions to reduce the time for compression interruptions. In order to reduce the chance of personnel exposure, save manpower, and improve the work efficiency of members of the resuscitation team, if the defibrillator is equipped with self-adhesive defibrillation pads, it is recommended to use pads for electrical defibrillation.

\section{Establishment of venous access}

The CA patients would experience peripheral circulation failure, peripheral vein collapse, and the medical worker involved in the rescue wear- ing strict protective isolation equipment in the FangCang is difficult to operate. The above factors will significantly reduce the success rate of puncture. Repeated attempts at venipuncture will affect the efficiency of the team's recovery. It is important to establish a reliable drug delivery channel as soon as possible. The bone marrow cavity infusion channel has the advantages of high first puncture success rate, short operation time, low technical difficulty, and high reliability (11). Therefore, in the rescue process, if the peripheral venipuncture is difficult or the attempt of the peripheral venipuncture fails twice, the establishment of an intramedullary infusion channel should be considered.

\section{Use of resuscitation drugs}

If the initial heart rhythm of a CA patient is a non-shockable rhythm, adrenaline should be given as soon as possible. The personnel in the FangCang wearing strict protective isolation equipment often lead to hypoxia, so the cause of this part of the worker with CA is hypoxia, and the initial heart rhythm is often non-shockable. The use of epinephrine in these patients is of great importance. Early administration of adrenaline can increase the chance of spontaneous circulation recovery. A standard dose of epinephrine $(1 \mathrm{mg})$ is still recommended and administered every 3-5 min (12).

\section{Patient transfer after resuscitation}

After the rescue, the patient return of spontaneous circulation (ROSC), under the command of the resuscitation team leader, coordinated with the out-of-vehicle rescue team as soon as possible to make the connection, and immediately prepared the transfer flat car, transfer ventilator, and ambulance during the rescue process. Make preparations for in-FangCang transfer, and transfer to a conditional hospital for further advanced life support treatment. Patients undergoing cardiopulmonary resuscitation for more than $30 \mathrm{~min}$ after CA and still have no spontaneous circulation recovery. Termination of cardiopulmonary resuscitation can be considered (13). In the rescue process, for patients who have not recovered 
spontaneously in the autonomous circulation, a comprehensive assessment should be performed. Once the recovery is predicted to be hopeless, the cardiopulmonary resuscitation should be terminated as soon as possible. The public security personnel in the FangCang will be responsible for contacting the relevant department outside the FangCang to prepare the corpse. In addition, minimizing the exposure time of medical worker in high-risk environments during transfers and reducing the chance of cross-infection among hospital worker.

CA is mainly manifested as a sudden stop of the patient's heartbeat, resulting in abnormal ejection function, disappearance of arterial pulse and heart sound, which is a common clinical critical and critical illness. The disease has the characteristics of rapid onset, rapid progress, severe symptoms, and high mortality. The workers in the FangCang hospital is unexposed and uninfected, and the occurrence of cardiac arrest is more special than that of ordinary patients. Patients and rescue personnel may be exposed and infected during the condition evaluation and rescue process.

Taken together, in order to reduce the risk of exposure and to avoid cross-infection in the hospital as much as possible, a high-quality resuscitation team in the FangCang must be established, a dedicated rescue room in the FangCang must be established, feasible rescue measures and procedures for cardiac arrest in the FangCang workers must be formulated, and personnel must be repeated exercises can ensure that the medical workers achieves the goal of zero infection.

\section{Conclusion}

As the rescue doctors of FangCang Hospital, we have put forward the rescue strategies for the workers who suffering from CA and shared our successful experiences in Wuhan FangCang of China with the doctors from the world. We aim to do our best to help doctors from countries around the world overcome the COVID-19 induced epidemic as soon as possible.

\section{Ethical considerations}

Ethical issues (Including plagiarism, informed consent, misconduct, data fabrication and/or falsification, double publication and/or submission, redundancy, etc.) have been completely observed by the authors.

\section{Acknowledgements}

This work was supported by grants from the Key Research and Development Program of Ningxia (Grant No.2019BEG03043).

\section{Conflict of interest}

The authors declare that there is no conflict of interest.

\section{References}

1. World Health Organization (2020), WHO Director-General's Remarks at the Media Briefing on 2019-nCoV on 11 February 2020. https://www.who.int/dg/speeches/detail/w ho-director-general-s-remarks-at-the-mediabriefing-on-2019-ncov-on-11-february-2020

2. Zhou P, Yang XL, Wang XG et al (2020). A pneumonia outbreak associated with a new coronavirus of probable bat origin. Nature, 579(7798):270-273.

3. World Health Organization (2020), Coronavirus Disease 2019 (COVID-19) Situation Report12. https://www.who.int/docs/defaultsource/searo/timor-leste/20-02-2020-tlssitrep-12-ncov-eng.pdf?sfvrsn=5a0b21c5_2

4. Narayan SM, Wang PJ, Daubert JP (2019). New concepts in sudden cardiac arrest to address an intractable epidemic: JACC state-of-the-art review. I Am Coll Cardiol, 73(1): 70-88.

5. Kronick SL, Kurz MC, Lin S et al (2015). Part 4: Systems of Care and Continuous Quality Improvement: 2015 American Heart Association Guidelines Update for Cardiopulmonary Resuscitation and Emergency Cardiovascular Care. Circulation, 2015,132:S397-413.

6. Kleinman ME, Brennan EE, Goldberger ZD et al. Part 5: Adult Basic Life Support and Car-

Available at: http://ijph.tums.ac.ir 
diopulmonary Resuscitation Quality: 2015 American Heart Association Guidelines Update for Cardiopulmonary Resuscitation and Emergency Cardiovascular Care. Circulation, 132:S41435.

7. Link MS, Berkow LC, Kudenchuk PJ et al (2015). Part 7: Adult Advanced Cardiovascular Life Support: 2015 American Heart Association Guidelines Update for Cardiopulmonary Resuscitation and Emergency Cardiovascular Care. Circulation, 2015,132:S444-64.

8. Panchal AR, Berg KM, Hirsch KG et al (2019). 2019 American Heart Association Focused Update on Advanced Cardiovascular Life Support: Use of Advanced Airways, Vasopressors, and Extracorporeal Cardiopulmonary Resuscitation during Cardiac Arrest: An Update to the American Heart Association Guidelines for Cardiopulmonary Resuscitation and Emergency Cardiovascular Care. Circulation, 2019,140:e881-e894.

9. Duff JP, Topjian AA, Berg MD et al (2020). 2019 American Heart Association Focused
Update on Pediatric Advanced Life Support: An Update to the American Heart Association Guidelines for Cardiopulmonary Resuscitation and Emergency Cardiovascular Care. Pediatrics, 145(1): pii: e20191361.

10. Tran K, Cimon K, Severn M et al (2012). Aerosol generating procedures and risk of transmission of acute respiratory infections to healthcare workers: a systematic review. PLoS One, 7:e35797.

11. Johnson M, Inaba K, Byerly S et al (2016). Intraosseous Infusion as a Bridge to Definitive Access. Am Surg, 82(10):876-880.

12. Andersen LW, Kurth T, Chase M et al (2016). Early administration of epinephrine (adrenaline) in patients with cardiac arrest with initial shockable rhythm in hospital: propensity score matched analysis. BMJ, 353:11577.

13. Wang L, Shi Y, Xiao T et al (2020). Chinese expert consensus on the perinatal and neonatal management for the prevention and control of the 2019 novel coronavirus infection (First Edition). Ann Transl Med, 8(3):47. 